\title{
A!
}

This is an electronic reprint of the original article.

This reprint may differ from the original in pagination and typographic detail.

Reentilä, Outi; Mattila, Marco; Knuuttila, Lauri; Hakkarainen, Teppo; Sopanen, Markku; Lipsanen, Harri

In-situ determination of nitrogen content in InGaAsN quantum wells

Published in:

Journal of Applied Physics

DOI:

$10.1063 / 1.2209772$

Published: 01/01/2006

Document Version

Publisher's PDF, also known as Version of record

Please cite the original version:

Reentilä, O., Mattila, M., Knuuttila, L., Hakkarainen, T., Sopanen, M., \& Lipsanen, H. (2006). In-situ determination of nitrogen content in InGaAsN quantum wells. Journal of Applied Physics, 100(1), 1-4. [013509]. https://doi.org/10.1063/1.2209772

This material is protected by copyright and other intellectual property rights, and duplication or sale of all or part of any of the repository collections is not permitted, except that material may be duplicated by you for your research use or educational purposes in electronic or print form. You must obtain permission for any other use. Electronic or print copies may not be offered, whether for sale or otherwise to anyone who is not an authorised user. 


\section{In situ determination of nitrogen content in InGaAsN quantum wells}

O. Reentilä, M. Mattila, L. Knuuttila, T. Hakkarainen, M. Sopanen, and H. Lipsanen

Citation: Journal of Applied Physics 100, 013509 (2006); doi: 10.1063/1.2209772

View online: http://dx.doi.org/10.1063/1.2209772

View Table of Contents: http://aip.scitation.org/toc/jap/100/1

Published by the American Institute of Physics

\section{$A|P|$ Journal of Applied Physics}

Save your money for your research. It's now FREE to publish with us no page, color or publication charges apply.

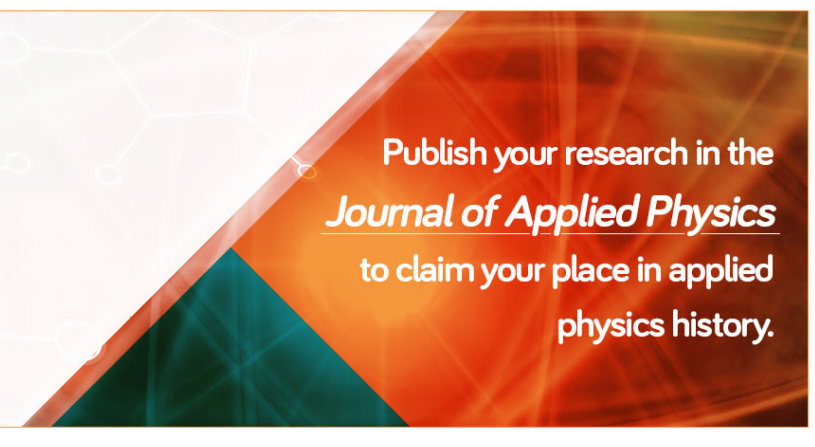




\title{
In situ determination of nitrogen content in InGaAsN quantum wells
}

\author{
O. Reentilä, a) M. Mattila, L. Knuuttila, T. Hakkarainen, M. Sopanen, and H. Lipsanen \\ Optoelectronics Laboratory, Micronova, Helsinki University of Technology, P.O. Box 3500, \\ FIN-02015 TKK, Finland
}

(Received 27 March 2006; accepted 21 April 2006; published online 7 July 2006)

\begin{abstract}
The growth of InGaAsN/GaAs multiple quantum well structures by metal-organic vapor phase epitaxy is monitored by in situ reflectometry. The nitrogen incorporation is found to depend superlinearly on the precursor flow and a threshold value for the flow is observed. By in situ measurements of the InGaAsN quantum well samples with a fixed indium content, the change in the reflectance during the quantum well growth is found to be linearly dependent on the quantum well nitrogen content. A model to determine the nitrogen content already during the growth is developed. Moreover, the field of application of in situ reflectance monitoring is extended from thick layers to thin layers, including quantum wells. (C) 2006 American Institute of Physics.
\end{abstract}

[DOI: 10.1063/1.2209772]

\section{INTRODUCTION}

The research of dilute nitrides has achieved a solid position in the research of compound semiconductors since Kondow et al. ${ }^{1}$ published their results concerning the effects of nitrogen on the properties of InGaAsN. It has been observed that the adjustment of the nitrogen content and the control of the material quality are demanding tasks both in metal-organic vapor phase epitaxy (MOVPE) and in molecular beam epitaxy (MBE). When growth processes are developed for these challenging materials the information obtained during the growth by in situ monitoring is all the more important. MOVPE and MBE systems both have the possibility of in situ monitoring the growth by nondestructive methods.

In situ reflectance measurements of MOVPE growth are fast, easy, and practical way to monitor the progress of crystal growth. They are commonly used to monitor the growth of thick layers of III-N materials; see, for example, Hardtdegen et al. $^{2}$ The in situ monitoring of the growth of thick GaAs and AlAs layers has also been studied. ${ }^{3}$ However, it is also possible to monitor the growth of thin layers, e.g., quantum wells (QWs), ${ }^{4}$ and the layers can even be partially analyzed during the growth. ${ }^{5}$ Here we use in situ reflectance measurements to monitor the MOVPE growth of InGaAsN quantum wells. Furthermore, a model to determine the QW nitrogen content during the growth is derived.

\section{EXPERIMENT}

The samples were fabricated by low-pressure close coupled showerhead MOVPE system at 100 torr pressure on semi-insulating (100) GaAs substrates using hydrogen $\left(\mathrm{H}_{2}\right)$ as a carrier gas. The total flow to the reactor was $61 / \mathrm{min}$. Trimethylindium (TMIn), trimethylgallium (TMGa), tertiarybutylarsine (TBAs), and dimethylhydrazine (DMHy) were used as precursors for indium, gallium, arsenic, and nitrogen, respectively. Two GaAs buffer layers, the first one at $660^{\circ} \mathrm{C}$

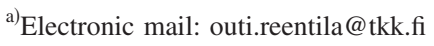

and the second at $575^{\circ} \mathrm{C}$ (thermocouple readings), were grown on the 350- $\mu$ m-thick substrate before the QW structure which consisted of four InGaAsN/GaAs QWs. The growth temperature of the $\mathrm{QW}$ structure was $575^{\circ} \mathrm{C}$. The QW and barrier thicknesses were approximately 8 and $15 \mathrm{~nm}$, respectively. No extra capping was used on top of the uppermost barrier layer. TMGa, TMIn, and TBAs molar flows to the reactor were kept constant for all the samples. TBAs/III molar flow ratio for all the QWs was 2.23 while the DMHy molar flow to the reactor was varied from 0 to $1680 \mu \mathrm{mol} / \mathrm{min}$ (DMHy/III molar flow ratios are $0-37$, $\mathrm{DMHy} / \mathrm{V}$ ratios are 0-0.94) to fabricate samples with different nitrogen contents.

In situ monitoring of the growth was realized with a normal incident reflectance setup using a halogen lamp as a light source. The rotation speed of $100 \mathrm{rpm}$ of the susceptor led to a sampling frequency of $1.7 \mathrm{~Hz}$. In our system, the reflected signal can be recorded at either 635 or $950 \mathrm{~nm}$. In this study, the reflectance was measured at $635 \mathrm{~nm}$.

The layer thicknesses, indium contents, and nitrogen contents of the QWs were determined by high-resolution $\mathrm{X}$-ray diffraction (XRD) measurements ( $\omega$ - $2 \theta$ configuration) and simulations together with photoluminescence (PL) measurements and band anticrossing (BAC) model. ${ }^{6}$ Combining the information obtained from these methods the nitrogen content of the samples was found to vary from $0 \%$ to $5 \%$. The indium content was 16.5\%-19\%. Both low temperature photoluminescence (LTPL) $(10 \mathrm{~K})$ and room temperature photoluminescence (RTPL) measurements were carried out to determine the luminescence energy and optical quality of the samples. Ex situ atmospheric pressure MOVPE annealing using TBAs to protect the surface from desorption was performed for $10 \mathrm{~min}$ at $700{ }^{\circ} \mathrm{C}$ to enhance the PL intensity of the samples. LTPL and RTPL measurements were performed for as-grown samples as well as for the annealed samples.

\section{RESULTS AND DISCUSSION}

Figure 1 shows the QW nitrogen content as a function of the DMHy molar flow. The nitrogen content has been re- 


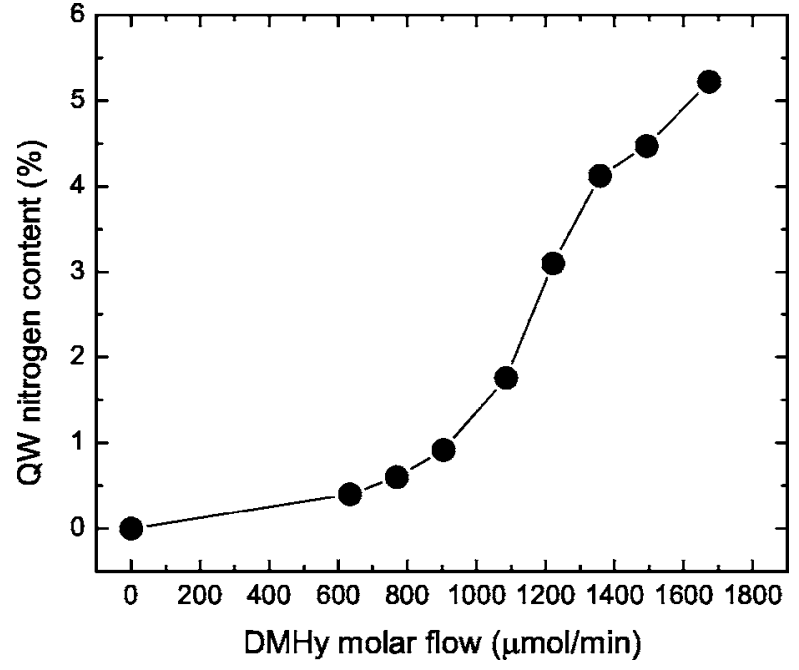

FIG. 1. QW nitrogen content as a function of DMHy flow. The curve indicates a threshold for nitrogen incorporation with a DMHy flow of about $850 \mu \mathrm{mol} / \mathrm{min}$.

ported earlier to have a superlinear dependence on the amount of nitrogen precursor in the vapor phase. ${ }^{7}$ A nonlinear correlation between the QW nitrogen content and the DMHy molar flow can also be seen in Fig. 1. In addition, a clear threshold for the nitrogen incorporation is observed as the DMHy flow is increased. The threshold DMHy flow appears to be around $850 \mu \mathrm{mol} / \mathrm{min}$. Below this value the nitrogen incorporation is relatively inefficient.

A typical XRD curve and a simulation fitted to that are shown in Fig. 2. XRD measurements were performed on as-grown samples to avoid any possible changes in the QW material and intermixing at the QW-barrier interfaces. In the simulated sample in Fig. 2, the QWs contained 19\% indium and $0.8 \%$ nitrogen. The quantum well thickness was $8.6 \mathrm{~nm}$. Good agreement between the measurement and simulation curves was found for all the samples. At large nitrogen contents the intensity of the satellite peaks in the XRD curve decreased which indicates a slight decrease of the crystal quality.

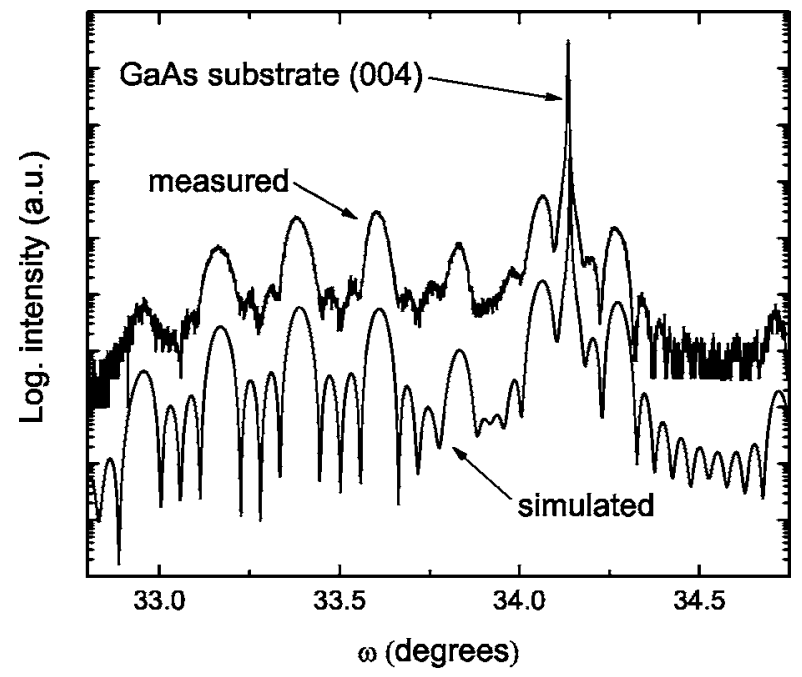

FIG. 2. Typical XRD measurement (upper curve) and a simulation result (lower curve) fitted to the measurement. For clarity, a vertical offset is added between the measured and simulated curves.

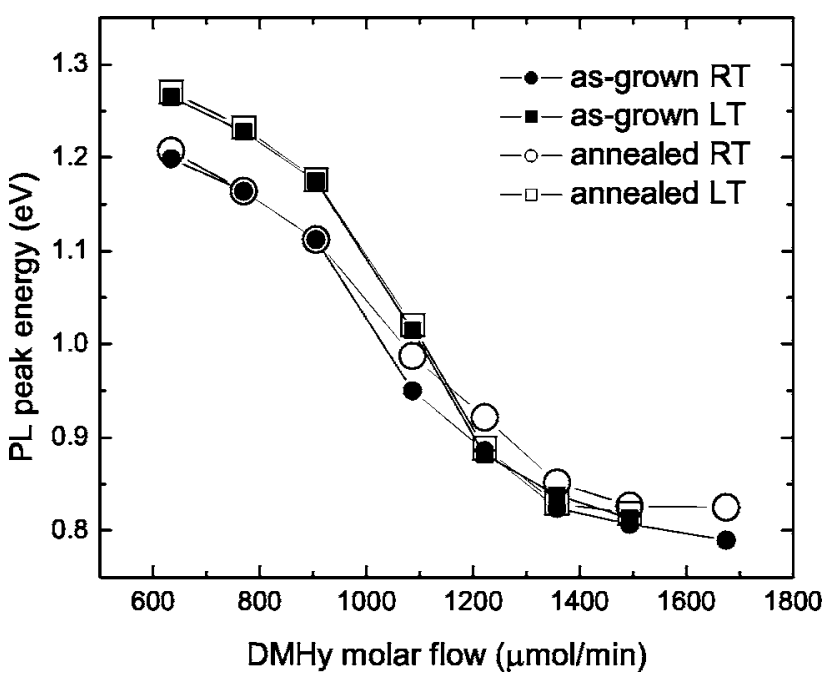

FIG. 3. RTPL and LTPL peak energies of as-grown and annealed samples as a function of the DMHy molar flow.

Figure 3 shows RTPL and LTPL peak energies from the as-grown and annealed samples as a function of the DMHy molar flow. The PL peak energies vary nonlinearly from 0.79 to $1.27 \mathrm{eV}$ depending on the DMHy flow and thus the QW nitrogen content (Fig. 1). To excite the as-grown samples sufficiently, a large pump power was needed. This, however, led to blueshift of the luminescence wavelength. Blueshift increased up to $35 \mathrm{~nm}$ as the luminescence wavelength increased. This is assumed to be caused by increasing compositional inhomogeneity within the quantum wells as the nitrogen content increases. No LTPL was observed from the sample grown with the largest DMHy molar flow.

Figure 4 shows a typical reflectance curve measured during the growth of the quantum well structure. QW growth regions (shaded in the figure) can be clearly distinguished from the barriers. The sample in Fig. 4 contained 5\% nitrogen and $16.5 \%$ indium in all four 7-nm-thick quantum wells separated by $16-\mathrm{nm}$-thick barrier layers. All the samples

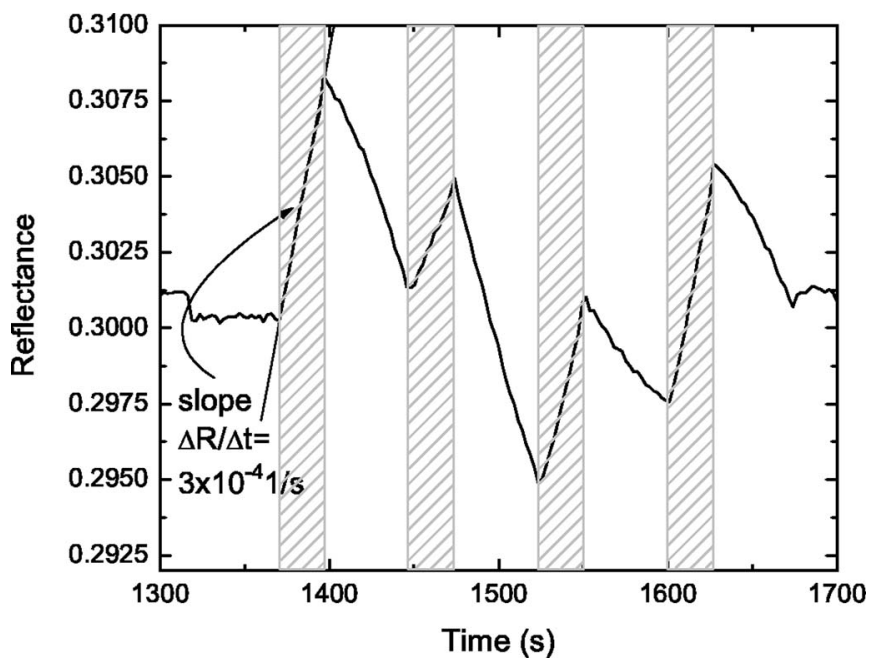

FIG. 4. Reflectance measured during the QW structure growth. QW growth regions are shaded and the unmarked regions between them are barrier growth regions. The linear fit to the reflectance measured during the growth of the first QW is also shown. 


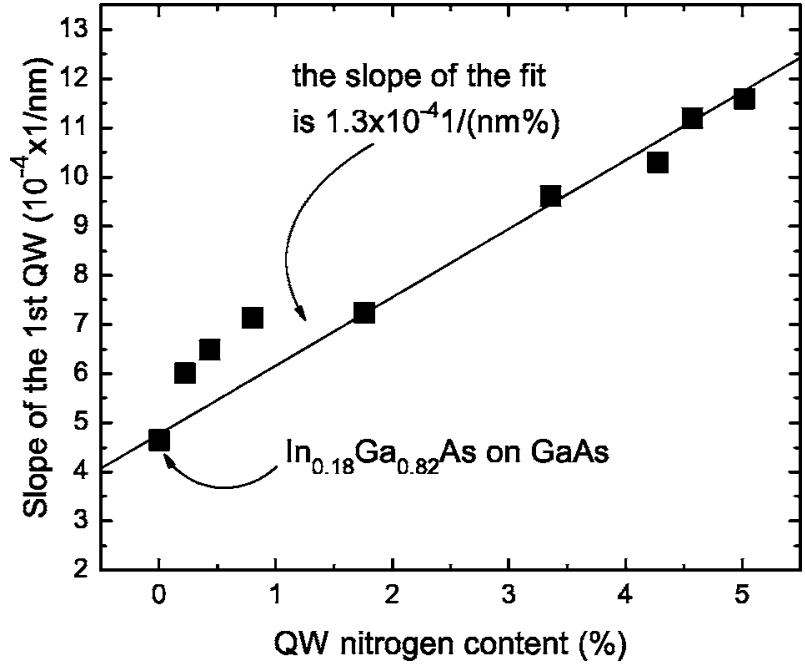

FIG. 5. Growth rate corrected slopes $\Delta R / \Delta d$ of the reflectance measured during the growth of the first QW as a function of the QW nitrogen content. A linear fit forced via the InGaAs data point gives a slope of 1.30 $\times 10^{-4} 1 /(\% \mathrm{~nm})$.

showed similar consistent behavior in in situ reflectance measurements. We have earlier observed that the deterioration of the crystal quality of the QW can be observed as changes in the in situ reflectance curve. ${ }^{5}$ Thus, the fact that all the samples fabricated here showed a similar shape of the in situ reflectance curves indicates a successful growth of the structures even with large nitrogen contents. The good crystalline quality was also confirmed by the XRD measurements.

The changes in the reflectance in Fig. 4 originate from the different complex refractive indices of GaAs and InGaAsN. Also, a change in temperature can cause variations in reflectance, but here the whole QW stack was grown at a constant temperature. Naturally, the refractive index of InGaAsN depends on the nitrogen and indium contents: the increase of both leads to a larger refractive index difference between GaAs and the QW. The reflectance increases as the $\mathrm{QW}$ is grown and decreases during the barrier growth. A linear fit to the reflectance measured during the growth of the first QW is shown in Fig. 4 and has a slope $\Delta R / \Delta t$ of 3.0 $\times 10^{-4} 1 / \mathrm{s}$. Because the difference of reflectance $\Delta R$ is linearly dependent on the layer thickness (as the first approximation when the layer is thin), the slope $\Delta R / \Delta t$ is linearly dependent on the growth rate. The first $\mathrm{QW}$ has been chosen to be the one of interest because of simplicity: below the first QW there is only a thick layer of GaAs, whereas below the subsequent QWs there are also the other QWs already grown.

Figure 5 shows the growth rate corrected reflectance slopes $\Delta R / \Delta d$ of the samples with different nitrogen contents in the QWs. The slopes in Fig. 5 are obtained in units of $(1 / \mathrm{nm})$ by taking the slopes $\Delta R / \Delta t$ in $(1 / \mathrm{s})$ and dividing them with the actual QW growth rates. After the growth rate correction the slope $\Delta R / \Delta d$ depends only on the composition of the quantum well. The slope increases linearly with the QW nitrogen content assuming that the QW indium content is constant. A linear fit forced via the InGaAs data point is shown in the figure with a slope of $1.30 \times 10^{-4} 1 /$
$(\% \mathrm{~nm})$. The deviations from the fit are assumed to be caused mostly by the slightly different QW indium contents of the samples. The samples grown with the smallest DMHy molar flows contain two percentage units more indium than the sample grown with the largest DMHy molar flow. As a result, the data points for the three samples with small nitrogen content remain a bit above the linear fit in Fig. 5.

In the light of these results we report that for a given indium content, the nitrogen content $y$ of the InGaAsN QW can be written as

$$
y=\frac{k_{\mathrm{InGaAsN}}-k_{\mathrm{InGaAs}}}{A},
$$

where $k_{i}$ is the reflectance slope $\Delta R / \Delta d$ from material $i$, and $A$ is a constant. $A$ can be determined from the linear fit to the growth rate corrected slope $\Delta R / \Delta d$ as a function of the QW nitrogen content. We expect this method to be suitable for any fixed indium content. For $\mathrm{In}_{0.18} \mathrm{Ga}_{0.82} \mathrm{AsN}, A$ is 1.30 $\times 10^{-4} 1 /(\% \mathrm{~nm})$ and $k_{\mathrm{InGaAs}}$ is $4.65 \times 10^{-4} 1 / \mathrm{nm}$. For a given indium composition, $k_{\text {InGaAs }}$ and $A$ are constants and, therefore, the nitrogen content can be determined straightforwardly from the in situ data.

In situ reflectance provides information about the sample composition and quality ${ }^{5}$ which makes it a fast and easy to use tool for characterizing dilute nitride QW samples already during the fabrication of the sample. We would like to point out that thick layers are not needed, but a layer thickness of a few nanometers is enough to enable the use of in situ reflectance to determine the composition. In addition to the dilute nitride samples investigated here and in Ref. 5, the method should be applicable to any material combination with different refractive indices. We are currently in the process of expanding the model to include all the QWs in the structure and not only the first QW. Then the homogeneity of the structure in the vertical direction, e.g., the similarity of the QWs in the multi-quantum-well (MQW) structure, could also be observed during the growth.

\section{CONCLUSION}

MOVPE growth of InGaAsN/GaAs MQW structures has been monitored using in situ reflectance measurements. XRD characterization together with PL measurements has been performed and the BAC model is utilized to determine the QW nitrogen content. Based on the measurements and simulations, a clear thresholdlike behavior of the QW nitrogen content as a function of DMHy molar flow was observed. Furthermore, the nitrogen content of the InGaAsN/GaAs quantum wells with a fixed indium content is found to be linearly dependent on the slope of the in situ reflectance measured during the quantum well growth. Based on these results, we have developed a method to determine the nitrogen content of quantum wells in situ from the reflectance curve measured during the growth in the MOVPE reactor. The model developed in this study is applicable to the typical nitrogen content range for dilute nitrides (from $0 \%$ to $5 \%$ ) and extends the field of application of the reflectance monitoring to include the growth of thin layers. 
${ }^{1}$ M. Kondow, K. Uomi, A. Niva, T. Kitatani, S. Watahiki, and Y. Yasawa, Jpn. J. Appl. Phys., Part 1 35, 1273 (1996).

${ }^{2}$ H. Hardtdegen, N. Kaluza, R. Schmidt, R. Steins, E. V. Yakolev, R. Talalaev, Y. N. Makarov, and J.-T. Zettler, Phys. Status Solidi A 201, 312 (2004).

${ }^{3}$ A. Rebey, M. Habchi, A. Bchetnia, and B. El Jani, J. Cryst. Growth 261 450 (2004)

${ }^{4}$ R. Lum, J. McDonald, J. Bean, J. Vandenberg, T. Pernell, S. Chu, A.
Robertson, and A. Karp, Appl. Phys. Lett. 69, 928 (1996).

${ }^{5}$ O. Reentilä, M. Mattila, M. Sopanen, and H. Lipsanen, J. Cryst. Growth 290, 345 (2006)

${ }^{6}$ W. Shan, W. Walukiewicz, J. Ager, E. Haller, J. Geisz, D. Friedman, J. Olson, and S. Kurtz, Phys. Rev. Lett. 82, 1221 (1999).

${ }^{7}$ T. Miyamoto, T. Kageyama, S. Makino, D. Schlenker, F. Koyama, and K. Iga, J. Cryst. Growth 209, 339 (2000). 